\title{
Aspiration, CTCAE
}

National Cancer Institute

\section{Source}

National Cancer Institute. Aspiration, CT CAE. NCI Thesaurus. Code C28245.

A disorder characterized by inhalation of solids or liquids into the lungs. 